\title{
Surgical management of hypotropia in congenital fibrosis of extraocular muscles (CFEOM) presented by pseudoptosis
}

This article was published in the following Dove Press journal:

Clinical Ophthalmology

19 December 2012

Number of times this article has been viewed

\author{
Hatem A Tawfik' \\ Mohammad A Rashad ${ }^{2}$ \\ 'Oculoplastic Service, ${ }^{2}$ Pediatric \\ Ophthalmology Service, \\ Ophthalmology Department, \\ Ain Shams University, Cairo, Egypt
}

Correspondence: Mohammad A Rashad Pediatric Ophthalmology Service, Ophthalmology Department, Ain Shams University, Othman Building, Ali Amen Street, Cairo, Egypt Tel +20 I22 3463327

Emailmahmad_r@yahoo.com
Purpose: To describe the demographics, characteristics, management pitfalls, and outcomes of pseudoptosis associated with congenital fibrosis of the extraocular muscles (CFEOM).

Methods: A retrospective review was performed of eight patients presenting with ptosis and hypotropia to oculoplastic service. All patients underwent full ocular evaluation and magnetic resonance imaging of brain and orbit. Five of these patients underwent stepwise correction of hypotropia by single-stage adjustable strabismus surgery (SSASS), followed by a frontalis sling if needed.

Results: Eight patients had congenital strabismus with severe ptosis and a positive forced duction test. There was a highly significant improvement from preoperative mean hypotropia angle of 30 prism diopters (PD) to $9 \mathrm{PD}$ mean postoperative angle $(P=0.006)$. Surgery for ptosis was not needed in $80 \%$ of eyes.

Conclusion: CFEOM involving both ptosis and hypotropia could be properly managed with the correct sequence of surgical steps. Proper vertical alignment by correction of hypotropia utilizing SSASS may alleviate the need for ptosis surgery.

Keywords: congenital fibrosis of extraocular muscles, CFEOM, single-stage adjustable suture surgery, SSASS, pseudoptosis

\section{Introduction}

In 1889, Gast ${ }^{1}$ described a case of congenital external ophthalmoplegia and was probably referring to hereditary extraocular muscles fibrosis. In 1912, Bradbourne ${ }^{2}$ described the condition with obviously autosomal dominant (AD) extraocular muscle fibrosis with ptosis. The syndrome is rare, but several studies, by Brown, ${ }^{3}$ Laughlin, ${ }^{4}$ Apt and Alexrod, ${ }^{5}$ and Harley et al, ${ }^{6}$ have described affected family members having a chin-up posture with ptosis and eyes fixed in a down-gaze.

Congenital fibrosis of the extraocular muscles (CFEOM) is a rare, congenital, and nonprogressive disorder with multiple extraocular muscle restrictions. Its diagnosis and classification is identified by clinical manifestations as well as by genetic evaluation. $^{7}$

CFEOM types 1 and 2 have bilateral ptosis and variable degrees of restriction of horizontal and vertical eye movements, which may be more severe in type 2. In CFEOM type 3 , the eyes may be not infraducted and may elevate above the midline. Ptosis may be absent or variable and may be unilateral. CFEOM types 1 and 3 are AD with full penetrance and variable expressivity; CFEOM type 2 is autosomal recessive (AR). ${ }^{8}$ CFEOM type 4 (Tukel syndrome) represents a CFEOM type 3 phenotype with postaxial oligodactyly or oligosyndactyly. ${ }^{9}$ 
CFEOM is challenging to treat for a number of reasons. First, because stepwise correction of strabismus should be performed before ptosis; second, surgery in CFEOM has unpredictable outcomes because of restrictive nature of the condition; third, the absence of Bell's phenomenon in CFEOM increases risk of exposure keratopathy. ${ }^{10}$

The present study tried to focus on surgical management of phenotype appearance rather than looking at genetic classification. This utilized the proper algorithm and resulted in near-total correction of hypotropia angle. We used the single-stage adjustable strabismus surgery (SSASS) technique. The details of correction of strabismus in CFEOM have not been mentioned in previous literature, and none of the few previous reports we reviewed used SSASS technique.

\section{Patients and methods}

The present study retrospectively reviewed seven Egyptian patients and one girl from Gaza who presented between 2008 and 2011 with congenital ptosis to oculoplastic service. They were referred to pediatric ophthalmology service as they were all noticed to have vertical eye movement misalignment.

\section{Inclusion criteria}

- Ptosis since birth.

- Hypotropia.

- Positive forced duction test (FDT).

- Unilateral and bilateral cases.

\section{Exclusion criteria}

- Negative FDT.

- History of orbital trauma.

- Acquired ptosis.

- Globe retraction with duction movements.

- Suspected or established diagnosis of myasthenia gravis or any other myopathy.

- Pigmentary retinopathy.

- Marcus Gunn jaw-winking phenomenon.

- Other causes of ptosis, such as brain stem or third nerve anomaly.

- Radiological anomaly of the brain or orbits.

All patients underwent proper history-taking, including family history of similar conditions and past ocular surgical history. Old pictures of the patients were requested, especially of those who had undergone surgery.

All patients also underwent assessment of eyelid position, margin reflex distance (MRD), levator function, vertical palpebral fissure height, and eyelid contour examination by an oculoplastic surgeon. The presence of lagophthalmos and Bell's phenomenon were also looked for.

All patients underwent full ophthalmologic assessment, including visual acuity (VA) if possible to assess, ocular motility examination, and abnormal head posture (AHP). They also underwent anterior segment, posterior segment, and neurological assessment of the cranial nerves. All patients underwent magnetic resonance imaging (MRI) of brain and orbits.

Patients and/or parents were informed about the nature of CFEOM and differential diagnosis like myopathy. They were informed about the performance of FDT before surgery under general anesthesia (GA). They were informed that surgery on extraocular muscles would be performed only in case of positive FDT. They were informed about the stepwise approach treating squint first, in which the squint surgery would be performed by a pediatric eye surgeon, after which, eyelid position would be judged by an oculoplastic surgeon 4 weeks after SSASS.

FDT was performed by holding the eye globe with two-toothed forceps at limbus and pulling the eye up to test the recti muscles. In cases of suspected inferior rectus (IR) fibrosis, the globe was held in the 3 and 9 o'clock positions and moved up-gaze.

If FDT was positive, the affected muscle was recessed using SSASS. Large recession of $12 \mathrm{~mm}$ or more was performed after release of all adhesions and fibrosis bands. During IR recession, the capsulopalpebral head was dissected from the surface of IR to safeguard against lower eyelid retraction. The patient was followed up on the same day as surgery, and at days 1,7 , and 30 postoperatively. If the patient needed adjustment, it was performed within the first week of surgery.

Adjustment was stopped when orthophoria was achieved or when maximum recession was performed to achieve best vertical alignment. After vertical alignment stability, the patient was referred back to an oculoplastic surgeon. The eyelid position, lagophthalmos, and corneal exposure were examined to assess the need for further eyelid surgery. If a patient accepted the second surgery, they were informed about the possibility of revision of eyelid position if needed.

Eyelid suspension was performed in only one patient, using conservative fascia lata technique to avoid keratopathy.

\section{Statistical analysis}

Data were analyzed with the Statistical Package for the Social Sciences (SPSS) version 16 (IBM Corporation, Armonk, NY). 
Data are presented as mean, SD. Paired sample $t$-test and Wilcoxon signed-rank test were used to compare two paired groups with nonparametric data. $P<0.05$ was considered significant.

\section{Results}

Patient ages ranged from 9 months to 35 years old with a mean age of 12.8 years. Seven patients were female; only one patient was male. All patients had had ocular deviation since birth.

Previous ptosis surgery was performed once in one patient (Figure 1), twice in one patient, and three times in one patient (Figure 2) before presentation.

Four patients had bilateral presentation (Figure 3) and four patients had unilateral involvement (Figures 1 and 2). Three patients declined surgery after information about the surgery and the outcome possibilities. Eight eyes of five patients who underwent SSASS had inferior rectus restrictive myopathy, severe ptosis with poor levator function and chin-up position. Lagophthalmos was present in 5/8 (62.5\%) of the patients preoperatively. Mean preoperative MRD in the five postoperative patients was $3 \pm 1 \mathrm{~mm}$.

Mild to moderate visual impairment was found in $7 / 8$ patients $(87.5 \%)$ secondary to ptosis and error of refraction. Based on clinical signs and patterns of inheritance, all patients were diagnosed as CFEOM. Mean preoperative angle of hypotropia in the five postoperative patients was $30 \pm 7$ prism diopter (PD) in the primary position.

Minimum recession of the inferior rectus was $12 \mathrm{~mm}$. Readjustment was needed in $3 / 5$ patients $(60 \%)$. Of the five patients operated on for IR recession, four (80\%) had postoperative orthophoria (Figures 4 and 5) in the primary position with correction of AHP (Figure 6). Only one patient with unilateral presentation had under-corrected hypotropia postoperatively, despite readjustment.

Mean postoperative angle of hypotropia was $9 \mathrm{PD}$ with a high statistically significant difference from the preoperative

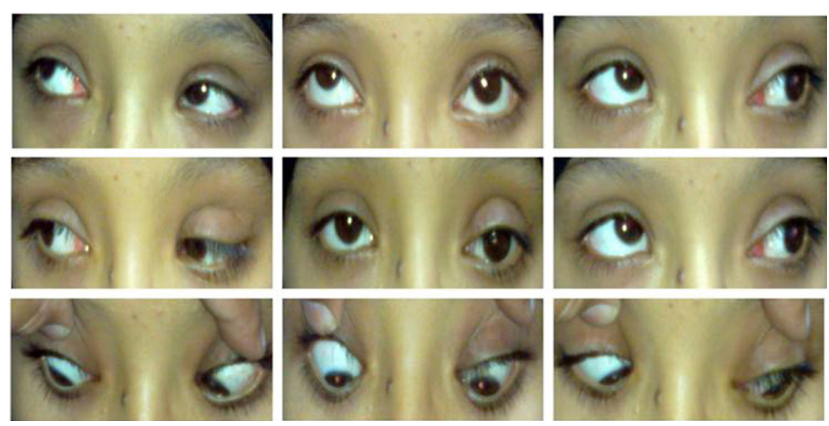

Figure I Preoperative presentation of Case 8 with left hypotropia and limited elevation in all gazes and one previous ptosis surgery.
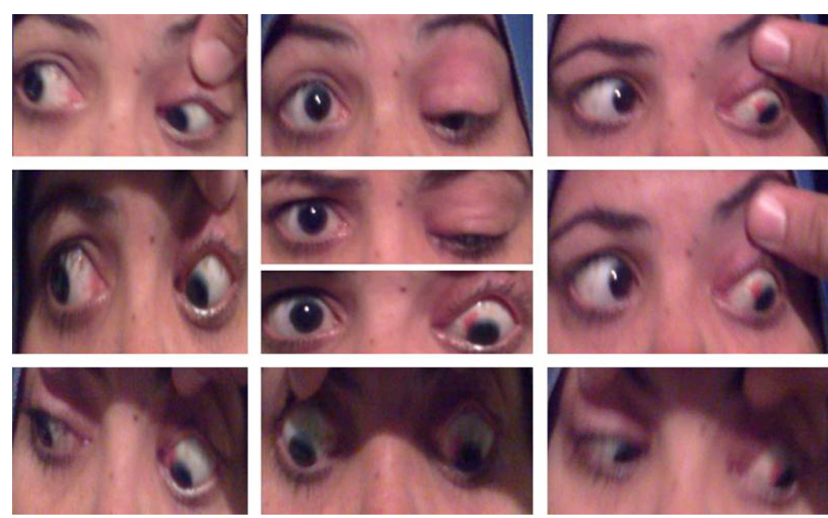

Figure 2 Preoperative presentation of Case 5 with left hypotropia and mismanaged ptosis by three sequential lid surgeries and lagophthalmos.

angle. Mean postoperative MRD was $1.5 \pm 1 \mathrm{~mm}$. Lagophthalmos was improved from mean preoperative $4 \mathrm{~mm}$ to mean postoperative $1.2 \mathrm{~mm}$. The difference was not statistically significant (Table 1).

Ptosis improved in four of five patients $(80 \%)$ and 6 of 8 eyes (75\%) (Figures 4 and 5). Only one of five patients (20\%) had postoperative persistent bilateral ptosis. He underwent sling operation for ptosis 1 month after SSASS (Figure 6). He had lid retraction with the need for relaxation of the eyelids to avoid corneal exposure effect.

Persistent lagohthalmos with exposure keratopathy was reported postoperatively in $2 / 5(20 \%)$ of operated patients and 2/8 (25\%) of operated eyes. Keratitis was found in one eye that had three previous successive ptosis surgeries prior to our SSASS (Figure 7), and in one eye of patient who underwent ptosis surgery following SSASS (Figure 6). All needed artificial tear drops and Thilo-Tears ${ }^{\circledR}$ at bed time. Only one patient needed a lid-lengthening procedure. Chinup position was corrected in $100 \%$ of operated patients.

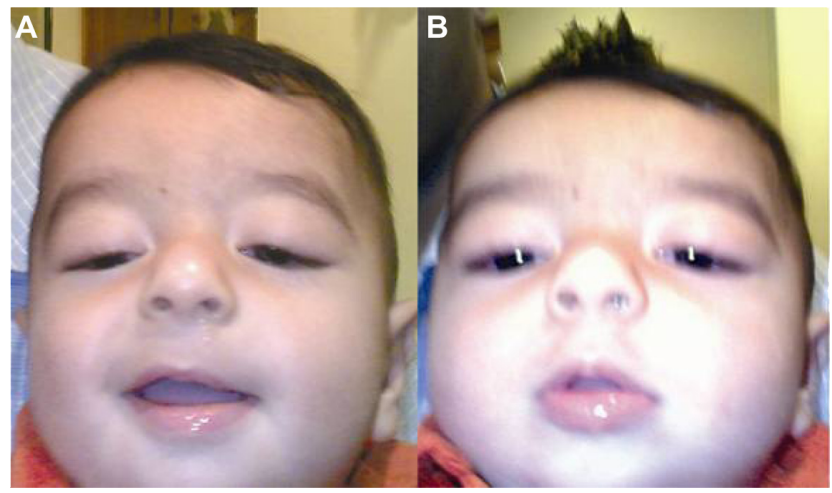

Figure 3 Preoperative presentation of bilateral hypotropia bilateral pseudoptosis (A) in Case 3, with chin-up head posture to fixate (B). 
Table I Comparison of pre- and postoperative parameters in 5 postoperative patients

\begin{tabular}{lllll}
\hline & Mean & SD & $\boldsymbol{t}$ & P-value \\
\hline Angle of deviation (PD) & & & & \\
$\quad$ Preoperative & 30 & 7.01 & 5.250 & $0.006^{*}$ \\
$\quad \begin{array}{l}\text { Postoperative } \\
\text { Lagophthalmos (mm) }\end{array}$ & 9 & 3.41 & & \\
$\quad$ Preoperative & 4.0 & 4.0 & 1.633 & 0.102 \\
$\quad \begin{array}{l}\text { Postoperative } \\
\text { MRD (mm) }\end{array}$ & 1.2 & 1.8 & & \\
$\quad$ Preoperative & 3 & 1 & 1.533 & 0.121 \\
$\quad$ Postoperative & 1.5 & 1 & & \\
\hline
\end{tabular}

Note: *Highly significant.

Abbreviations: PD, prism diopters; MRD, margin reflex distance.

Amblyopia was improved in 7/8 (87.5\%) of the operated eyes. The only eye that did not improve was that of a 35 -year-old.

\section{Discussion}

CFEOM is the term used to describe a large spectrum of several rare inherited strabismus syndromes that manifest as congenital restrictive ophthalmoplegia in one or more fields of gaze. ${ }^{10}$ The spectrum includes unilateral as well as bilateral cases, and one muscle (IR) as well as more than one muscle affection.

Accordingly, we included unilateral and bilateral cases and isolated muscle affection. The hallmark of CFEOM is reduced ${ }^{11,12}$ movement of the eye away from the direction of action of the affected muscle, confirmed by FDT. ${ }^{13}$ On this basis, the present study took into consideration confirmed diagnosis of CFEOM by limitation or restriction of FDT under anesthesia - an important key step in the planned stepwise management process. The excluded cases of negative FDT helped to discount eyes with similar conditions like myasthenia gravis, congenital third nerve palsy, double elevator palsy, and chronic progressive external ophthalmoplegia. We included only eyes with ptosis to exclude differential diagnosis list cases like Brown, Mobius, and

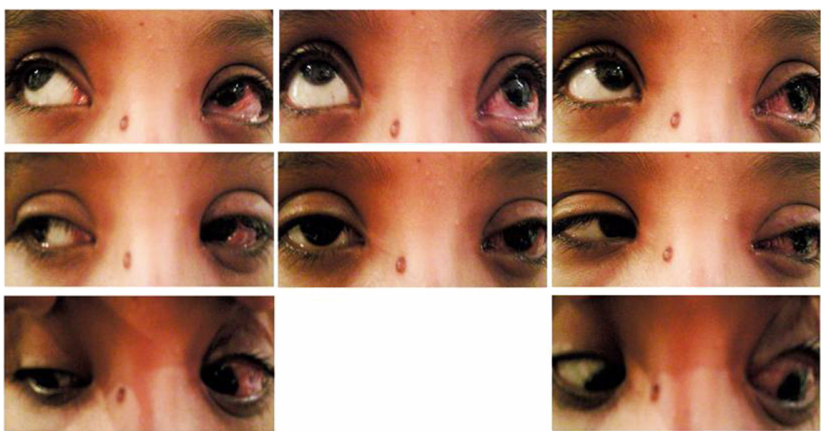

Figure 4 Postoperative pictures of Case 8 (I week after single-stage adjustable strabismus surgery) with residual ptosis due to lid edema.

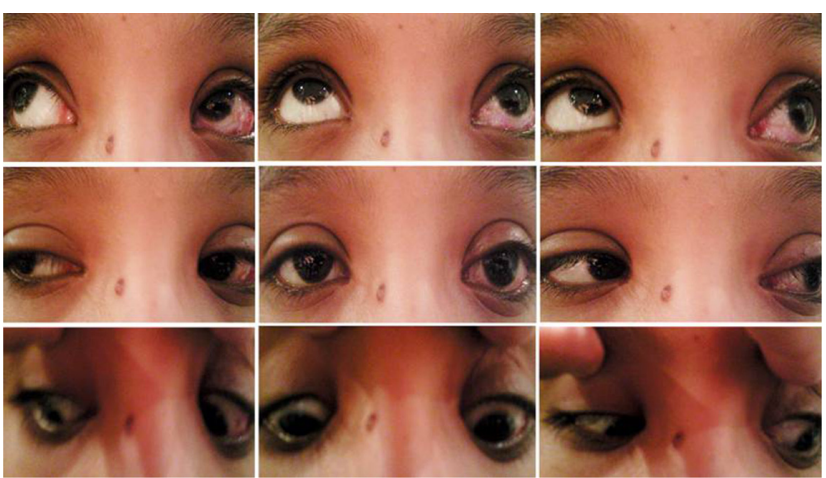

Figure 5 Postoperative pictures of Case 8 (I month after surgery) with less lid edema and improved up-gaze of left eye.

Duane syndromes. This differential diagnosis was listed by Cooymans et al. ${ }^{14}$ The strict inclusion and exclusion criteria of our study helped us to diagnose CFEOM based on clinical assessment (phenotypical) rather than on genetic assessment (genotypical). This was similar to Cooymans et al's ${ }^{14}$ description of their cases as CFEOM type 2 based on clinical assessment only without genetic study.

The overlap in clinical features between types of CFEOM may mean that it is necessary to perform genetic evaluation to differentiate between types. CFEOM type 1 most commonly results from mutations in the $K I F 21 A$ gene. CFEOM type 2 results from mutations in $\mathrm{PHOX} 2 \mathrm{~A}$ gene. CFEOM type 3 can be due to mutations in the TUBB3 gene in chromosome 16, $K I F 21 A$ gene in chromosome 12 , or unknown gene locus in

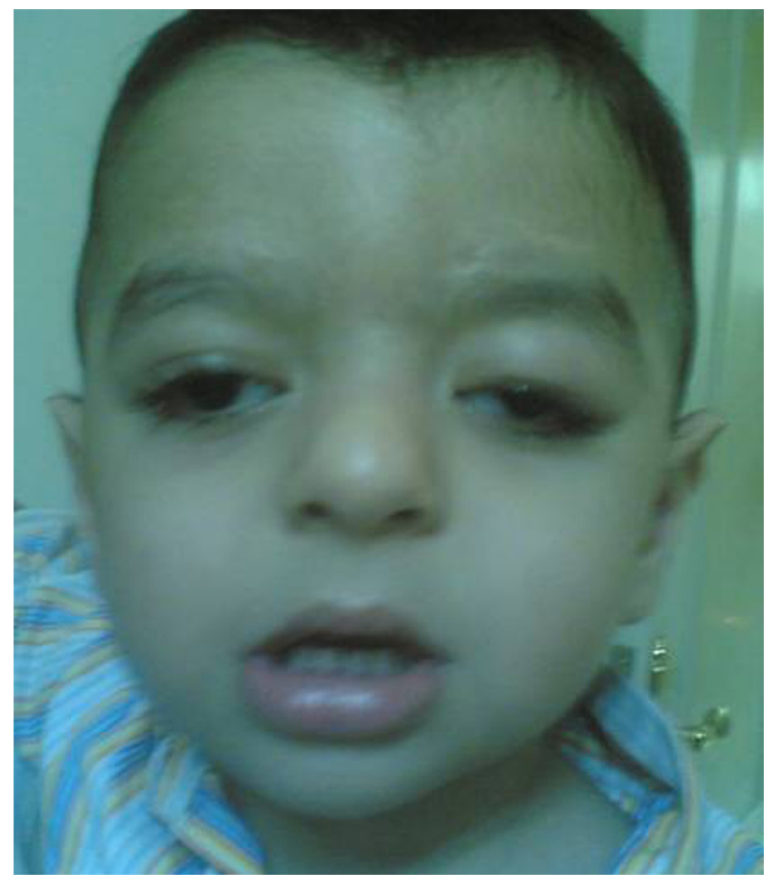

Figure 6 Postoperative picture of Case 3 after correction of hypotropia and conservative sling operation showing improvement of chin-up position. 


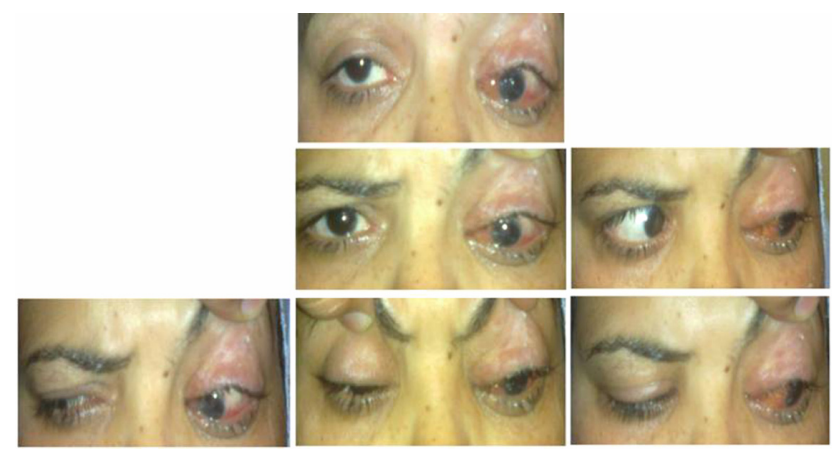

Figure 7 Postoperative pictures of Case 5 (I month after single-stage adjustable strabismus surgery) with maximum IR recession showing limited left-eye elevation, under-corrected hypotropia, residual ptosis, and lagophthalmos due to shortened lid.

chromosome 13. This means that the CFEOM phenotype include at least 4 known genetic mutations ${ }^{15}$ that prevent normal development of cranial nerves III and/or IV and their nuclei in different patterns. CFEOM results in an absence of superior division of the oculomotor nerve. CFEOM type 2 results in an absence of motor neurons in oculomotor and trochlear nuclei. CFEOM type 3 results in a variable developmental anomaly of the oculomotor nerve, more in the superior branch than in the inferior branch. ${ }^{16}$ This evidence suggests that the fibrotic changes in muscles are secondary to defective innervation of the muscles during development.

The term "congenital cranial dysinnervation disorders" (CCDDs) was coined to refer to the innervation disorders of the extraocular muscles. The various forms of CFEOM are included in the CCDDs. CCDDs are primarily due to neurogenic disturbances of brain stem or cranial nerve development. ${ }^{17}$

The term CCDD has been proposed as a substitute for the traditional concept of CFEOM, based on mounting genetic, neuropathologic evidence suggesting that many, if not all, of these disorders result primarily from neurological rather than muscle maldevelopment. A review of pertinent articles from 2003 to 2010 describing CCDD variants identified that a total of seven disease genes and ten phenotypes fall under the CCDD umbrella. ${ }^{17}$

With all of these genotypic variants, the diagnosis can be made clinically from the unique eye movement pattern and severe ptosis confirmed by positive FDT. ${ }^{10}$ This supports the methodology used in the present study, which was dependent mainly on clinical manifestations. However, we can describe our cases as CFEOM type 2 based on patterns of inheritance, CFEOM type 2 is described as being autosomal recessive, meaning that none of the ancestry had a similar disorder. ${ }^{8}$ This was reported in our study patients, as none of them had positive family history in any ancestor. CFEOM type 1 transmission is autosomal dominant with familial incidence.
CFEOM type 3 is autosomal dominant with incomplete penetrance and variable expression. ${ }^{12}$

Our report of our Egyptian cases as CFEOM type 2 corresponds with similar descriptions in Turkey, Saudi Arabia, and Iran. At the same time, CFEOM type 1 and CFEOM type 3 have been reported worldwide. ${ }^{14}$

Further confirmation by genetic evaluation did not affect the decision of surgery in our study.

The present study reported highly significant improvement of vertical hypotropia angle by a single procedure with adjustable suture technique. This technique, although not reported in CFEOM, has been reported in restrictive strabismus. ${ }^{18}$ This technique resulted in significant improvement as evidenced by the need for readjustment in $60 \%$ of the postoperative patients. This means that success rate would be only $40 \%$ of postoperative patients if SSASS was not used. This could explain the different results in Taylor and Gregson, who stated that very large recessions may be ineffective in CFEOM with very unpredictable outcomes. ${ }^{13}$

Full correction of the hypotropia angle resulted in improvement of lid position in $80 \%$ of the operated patients without need for further eyelid surgery.

The ptosis surgery was needed in only one patient (20\%) of our postoperative patients. This fact confirms that ptosis was mainly pseudoptosis where eyelids follow eyes tied in the down-gaze. Thus, pseudoptosis due to CFEOM should be considered in differential diagnosis of ptosis to prevent surgical mismanagement. This was different from the report of Liu et al, ${ }^{19}$ who described bilateral ptosis surgery in seven patients with ptosis in CFEOM, with no reported details of vertical alignment. Liu et al also reported two of their cases as having had two previous strabismus surgeries. They did not describe the use of SSASS. They also reported two of their cases as having had previous ptosis surgery before strabismus surgery without details about any residual misalignment. They mentioned in their technique for ptosis surgery that lids were left just closed at the end of surgery to overcome absent Bell's phenomenon. We differed by addressing the problem primarily by correcting the vertical misalignment almost totally, which may explain the need for ptosis surgery in only one of our patients. We had 2/8 (25\%) of operated eyes having exposure keratitis that may be due to the fact that CFOEM exhibit little or no Bell's phenomenon. Of these two eyes one had preoperative 3 ptosis surgeries before our SSASS and one eye had ptosis surgery after SSASS. This further confirms that ptosis surgery does not relieve the main problem in a patient with CFEOM. However, we had one case of unilateral dense corneal scar after 
persistent corneal ulceration, even when lid-lengthening procedure was performed. This may be explained by anterior segment ischemia. Wong and Jampolsky ${ }^{20}$ noticed that anterior ciliary circulation to the eye may be aberrant in some children with CFEOM. ${ }^{20}$ Operating even on one muscle may be associated with ischemia. Therefore, an examination of all muscle vasculature may be advisable before detaching any muscle. We also reported one of our cases having lower limb weakness with suspected myopathy. Reported cases of CFEOM showed associations that included musculoskeletal abnormalities. $^{21}$

\section{Conclusion}

Genetic evaluation of CFEOM may help to differentiate between types. However, the genotypic spectrum is still wide and enlarging under the CCDD term; therefore, diagnosis of CFEOM can be based on strict clinical criteria.

CFEOM remains an exceedingly rare phenomenon. However, it should be considered in differential diagnosis of ptosis by plastic and ophthalmic plastic surgeons, especially with vertical muscle misalignment dating since birth. The algorithm of management should be directed to proper vertical alignment first utilizing SSASS. Further eyelid surgery should be included in patient expectations although it may be not needed after SSASS.

\section{Disclosure}

This paper was presented in part at the European Society of Ophthalmic Plastic and Reconstructive Surgery Annual Meeting, Lucerne, Switzerland, September 2008. The authors report no conflicts of interest in this work.

\section{References}

1. Gast R. Ein Fall von ophthalmoplegia bilateralis exterior congenita. [One case of congenital external bilateral ophthalmoplegia]. Klin Monatsble Augenheilkd. 1889;27:214-217. German.

2. Bradburne AA. Hereditary ophthalmoplegia in five generations. Trans Opthalmol Soc UK. 1912;32:142-153.

3. Brown HW. Congenital muscle anomalies. In: Allen JH, editor. Strabismus Ophthalmic Symposium. St Louis: Mosby; 1950:229-233.

Clinical Ophthalmology

\section{Publish your work in this journal}

Clinical Ophthalmology is an international, peer-reviewed journal covering all subspecialties within ophthalmology. Key topics include: Optometry; Visual science; Pharmacology and drug therapy in eye diseases; Basic Sciences; Primary and Secondary eye care; Patient Safety and Quality of Care Improvements. This journal is indexed on Submit your manuscript here: http://www.dovepress.com/clinical-ophthalmology-journal
4. Laughlin RC. Congenital fibrosis of extraocular muscles; a report of six cases. Am J Ophthalmol. 1956;41:432-438.

5. Apt L, Axelrod RN. Generalized fibrosis of the extraocular muscles. Am J Ophthalmol. 1978;85:822-829.

6. Harley RD, Rodrigues MM, Crawford JS. Congenital fibrosis of extraocular muscles. J Pediatr Ophthalmol Strabismus. 1978;15:346-358.

7. Flaherty MP, Grattan-Smith P, Steinberg A, Jamieson R, Engle EC. Congenital fibrosis of extraocular muscles associated with cortical dysplasia and maldevelopment of the basal ganglia. Ophthalmology. 2001;108:1313-1322.

8. Yazdani A, Traboulsi EI. Classification and surgical management of patients with familial and sporadic forms of congenital fibrosis of the extraocular muscles. Ophthalmology. 2004;111:1035-1042.

9. Tukel T, Uzumcu A, Gezer A, et al. A new syndrome, congenital extraocular muscle fibrosis with ulnar hand anomalies, maps to chromosome 21qter. $J$ Med Genet. 2005;42(5):408-415.

10. Jaafar MS, Trabulsi EI. Congenital fibrosis of the extraocular muscles. In: Rosenbaum AL, Santiago AP, editors. Clinical Strabismus Management: Principles and Surgical Techniques. WB, Philadelphia: Saunders Company; 1999.

11. Engle EC, McIntosh N, Yamada K, et al. CFEOM1, the classic familial form of congenital fibrosis of the extraocular muscles, is genetically heterogeneous but does not result from mutations in ARIX. BMC Genet. 2002;3:3.

12. Doherty EJ, Macy ME, Wang SM, et al. CFEOM3: a new extraocular congenital fibrosis syndrome that maps to 16q24.2-q24.3. Invest Ophthalmol Vis Sci. 1999;40:1687-1694.

13. Taylor DSI, Gregson R. Congenital fibrosis syndromes. In: Good WV, Hoyt CS, editors. Strabismus Management. Newton, MA: ButterworthHeinemann; 1996:203-209.

14. Cooymans P, Al-Zuhaibi S, Al-Senawi R, Ganesh A. Congenital fibrosis of the extraocular muscles. Oman J Ophthalmol. 2010;3(2):70-74.

15. Khan AO, Khalil DS, Al Sharif LJ, Al-Ghadhfan FE, Al Tassan NA. Germline mosaicism for KIF21A mutation (p.R954L) mimicking recessive inheritance for congenital fibrosis of the extraocular muscles. Ophthalmology. 2010;117(1):154-158.

16. Lim KH, Engle EC, Demer JL. Abnormalities of the oculomotor nerve in congenital fibrosis of the extraocular muscles and congenital oculomotor palsy. Invest Ophthalmol Vis Sci. 2007;48:1601-1606.

17. Oystreck DT, Engle EC, Bosley TM. Recent progress in understanding congenital cranial dysinnervation disorder. J Neuroophthalmol. 2011;31(1):69-77.

18. Sharma P, Reinecke DR. Single-stage adjustable strabismus surgery for restrictive strabismus. JAAPOS. 2003;7(5):358-362.

19. Liu C, Ohri R, Frongia G, Collin R. Surgical correction of ptosis in ocular fibrosis syndrome. Br J Ophthalmol.1994;78:271-274.

20. Wong GY, Jampolsky A. Agenesis of three horizontal rectus muscles. Ann Ophthalmol. 1974;6:909-915.

21. Kishore K, Kumar H. Congenital ocular fibrosis with musculoskeletal abnormality: a new association. J Pediatr Ophthalmol Strabismus. 1991;28:283-286.
PubMed Central and CAS, and is the official journal of The Society of Clinical Ophthalmology (SCO). The manuscript management system is completely online and includes a very quick and fair peer-review system, which is all easy to use. Visit http://www.dovepress.com/ testimonials.php to read real quotes from published authors. 